\title{
Probability Distributions of Natural Frequencies of Uncertain Dynamic Systems
}

\author{
Sharif Rahman* \\ The University of Iowa, Iowa City, Iowa 52242 \\ DOI: $\underline{10.2514 / 1.42720}$
}

\begin{abstract}
This article presents a polynomial dimensional decomposition method for calculating the probability distributions of random eigenvalues commonly encountered in dynamic systems. The method involves a hierarchical decomposition of a multivariate function in terms of variables with increasing dimensions, a broad range of orthonormal polynomial bases consistent with the probability measure for a Fourier-polynomial expansion of component functions, and an innovative dimension-reduction integration for calculating the expansion coefficients. The new decomposition does not require sample points, yet it generates a convergent sequence of lower-variate estimates of the probability distributions of eigensolutions. Numerical results, including frequency distributions of a piezoelectric transducer, indicate that the decomposition method developed provides accurate, convergent, and computationally efficient estimates of the tail probabilistic characteristics of eigenvalues.
\end{abstract}

\begin{tabular}{|c|c|}
\hline & \\
\hline $\boldsymbol{A}_{j}$ & $=j$ th random coefficient matrix \\
\hline $\mathcal{B}^{N}, \mathcal{L}_{2}$ & $\begin{array}{l}=\text { Borel } \sigma \text { field on } \mathbb{R}^{N} \text { and Hilbert space, } \\
\text { respectively }\end{array}$ \\
\hline$C_{i_{1} \cdots i_{S} j_{1} \cdots j_{S}}$ & $=$ coefficient for $S$-variate component function \\
\hline$c, \Sigma_{X}$ & $=$ mean and covariance of $\boldsymbol{X}$, respectively \\
\hline & $=$ expectation operator \\
\hline$f$ & $=$ general nonlinear function \\
\hline$f_{i}\left(x_{i}\right)$ & $=$ marginal probability density of $X_{i}$ \\
\hline$f_{X}(\boldsymbol{x})$ & $=$ joint probability density of $\boldsymbol{X}$ \\
\hline$K(X)$ & $=$ random stiffness matrix \\
\hline$K_{i}(\boldsymbol{X})$ & $=i$ th random stiffness of spring \\
\hline$k^{*}$ & $=$ truncation parameter \\
\hline$L$ & $=$ size of random coefficient matrices \\
\hline$m$ & $=$ mass matrix \\
\hline$m$ & $=$ degree of orthonormal polynomial basis \\
\hline$N$ & $=$ number of random variables \\
\hline$n$ & $=$ number of Gaussian quadrature points \\
\hline$R$ & $=$ positive integer ranging from $S$ to $N$ \\
\hline $\mathbb{R}^{N}, \mathbb{C}^{N}$ & $\begin{array}{l}=N \text {-dimensional real and complex vector spaces, } \\
\text { respectively }\end{array}$ \\
\hline$S$ & $=$ positive integer ranging from 1 to $N$ \\
\hline $\boldsymbol{X}$ & $=$ input random vector \\
\hline$X_{i}$ & $=i$ th random variable \\
\hline$\alpha_{i j}$ & $\begin{aligned}= & \text { coefficient for univariate component function } \\
& \text { of } \lambda\end{aligned}$ \\
\hline $\begin{array}{l}\beta_{i_{1} i_{2} j_{1} j_{2}} \\
\lambda(\boldsymbol{X})\end{array}$ & $\begin{array}{l}=\text { coefficient for bivariate component function of } \lambda \\
=\text { random eigenvalue function }\end{array}$ \\
\hline$\gamma_{i_{1} i_{2} i_{3} j_{1} j_{2} j_{3}}$ & $\begin{aligned}= & \text { coefficient for trivariate component function } \\
& \text { of } \lambda\end{aligned}$ \\
\hline & $=$ imaginary part of $i$ th component of $\lambda$ \\
\hline & $=S$-variate component function of $\lambda$ \\
\hline & $=$ real part of $i$ th component of $\lambda$ \\
\hline$\hat{\lambda}_{R}(\boldsymbol{X})$ & $=R$-variate approximation of $\lambda$ \\
\hline$\tilde{\lambda}_{S}(\boldsymbol{X})$ & $=S$-variate polynomial approximation of $\lambda$ \\
\hline & $\begin{aligned}= & \text { first coefficient of polynomial decomposition } \\
& \text { of } \lambda\end{aligned}$ \\
\hline
\end{tabular}

Received 12 December 2008; revision received 19 February 2009; accepted for publication 22 February 2009. Copyright (C) 2009 by Sharif Rahman. Published by the American Institute of Aeronautics and Astronautics, Inc., with permission. Copies of this paper may be made for personal or internal use, on condition that the copier pay the $\$ 10.00$ per-copy fee to the Copyright Clearance Center, Inc., 222 Rosewood Drive, Danvers, MA 01923; include the code 0001-1452/09 \$10.00 in correspondence with the CCC.

*Department of Mechanical and Industrial Engineering, 2140 Seamans Center; rahman@engineering.uiowa.edu. $\begin{array}{ll}\psi_{i j} & =j \text { th univariate polynomial basis for } i \text { th variable } \\ (\Omega, \mathcal{F}, P) & =\text { probability space of } \boldsymbol{X} \\ \left(\Omega_{i}, \mathcal{F}_{i}, P_{i}\right) & =\text { probability space of } X_{i}\end{array}$

\section{Introduction}

$\mathbf{T}$ HE evaluation of natural frequencies and mode shapes of uncertain dynamic systems requires the solution of random eigenvalue problems, comprising stochastic matrix, differential, or integral operators. Classical methods for solving random eigenvalue problems are dominated by the perturbation method [1], a longstanding staple, but no longer considered state of the art as it is limited to problems with small uncertainties or small nonlinearities. Methods other than the perturbation method include the iteration method [1], the Ritz method [2] , the crossing theory [3] basis [4] , the asymptotic method []], the polynomial chaos expansion $[\overline{6}]$, and the recently developed dimensional decomposition method $[7,8]$. However, most existing methods delve into calculating the statistical moments of eigensolutions. A few of these methods also address how to calculate probability distributions of eigenvalues, for example, by fitting classical distribution functions from predicted second- or higher-order moments [5], but the resultant tail probabilistic characteristics, highly important for reliability analysis and design, have not been adequately scrutinized.

Recently, the author developed a dimensional decomposition method for calculating moments and probability distributions of real[7] or complex-valued [8] eigensolutions. However, the existing decomposition method requires a reference point, commonly assumed to be the mean value of the random input and sample points surrounding that reference point. Based on these sample points, deterministic calculations of eigenvalues, either exactly or numerically, are conducted to generate Lagrangian interpolations of various component functions embedded in the decomposition. There are two weaknesses in this procedure. First, the decomposition constructed above depends on the selected reference point, which, if improperly selected, can spoil the approximation. Second, and more important, the sample points are vaguely selected with no strict guidelines. If an input variable is strictly positive or strictly negative, or follows a probability density with compact support, the resultant sample points may fall outside the physical domain; in this case, existing decomposition methods may produce unrealistic sample properties of a random eigensolution. Therefore, alternative means of approximating the component functions by dropping the sample points altogether are desirable.

This paper presents a polynomial dimensional decomposition method for calculating the probability distributions of random eigenvalues frequently encountered in dynamic systems. The 
method is based on 1) a hierarchical decomposition of a multivariate function in terms of variables with increasing dimensions, 2) a broad range of orthonormal polynomial bases consistent with the probability measure for a Fourier-polynomial expansion of component functions, and 3) an innovative dimension-reduction integration for calculating the expansion coefficients. Section II formally defines the random eigenvalue problem, including citations of a few prominent applications. Section III describes the polynomial decomposition method, comprising multivariate function decomposition, Fourierpolynomial expansion, and dimension-reduction integration, and then discusses computational effort. Three numerical examples illustrate the accuracy, convergence, and computational efficiency of the proposed method in Sec. IV. Finally, the conclusions are drawn in Section V.

\section{Random Eigenvalue Problems in Dynamics}

Let $(\Omega, \mathcal{F}, P)$ be a complete probability space, where $\Omega$ is a sample space, $\mathcal{F}$ is a $\sigma$ field on $\Omega$, and $P: \mathcal{F} \rightarrow[0,1]$ is a probability measure. Let $\mathbb{R}^{N}$ and $\mathbb{C}^{N}$ be $N$-dimensional real and complex vector spaces, respectively, and $\mathbb{R}^{N \times N}$ a set of all $N \times N$, real-valued matrices. With $\mathcal{B}^{N}$ representing a Borel $\sigma$ field on $\mathbb{R}^{N}$ and $\mathbb{E}$ the expectation operator on $(\Omega, \mathcal{F}, P)$, consider an $\mathbb{R}^{N}$-valued, independent, input random vector $\left\{\boldsymbol{X}=\left\{X_{1}, \ldots, X_{N}\right\}^{T}:(\Omega, \mathcal{F}) \rightarrow\right.$ $\left.\left(\mathbb{R}^{N}, \mathcal{B}^{N}\right)\right\}$, which has mean $c:=\mathbb{E}[X] \in \mathbb{R}^{N}$, covariance matrix $\Sigma_{\boldsymbol{X}}:=\mathbb{E}\left[(\boldsymbol{X}-\boldsymbol{c})(\boldsymbol{X}-\boldsymbol{c})^{T}\right] \in \mathbb{R}^{N \times N}$, and joint probability density function $f_{X}(\boldsymbol{x})=\prod_{i=1}^{i=N} f_{i}\left(x_{i}\right)$, where $f_{i}\left(x_{i}\right)$ is the marginal probability density function of $X_{i}$ defined on the probability triple $\left(\Omega_{i}, \mathcal{F}_{i}, P_{i}\right)$. In most dynamic systems, the vector $X$ represents uncertainties in material parameters (e.g., mass, damping, stiffness), geometry (e.g., size, shape, topology), and constraints (e.g., initial and boundary conditions).

Consider a family of $L \times L$, real-valued, random coefficient matrices $\boldsymbol{A}_{j}(\boldsymbol{X}) \in \mathbb{R}^{L \times L}, j=1, \ldots, J$, where $J$ is a positive integer and a general nonlinear function $f$. The probabilistic characteristics of $\boldsymbol{A}_{j}(\boldsymbol{X})$ can be derived from the known probability law of $\boldsymbol{X}$. A nontrivial solution of

$$
f\left(\lambda(X) ; A_{1}(X), \ldots, A_{J}(X)\right) \phi(X)=\mathbf{0}
$$

if it exists, defines the random eigenvalue $\lambda(\boldsymbol{X}) \in \mathbb{R}$ or $\mathbb{C}$ and the random eigenvector $\phi(X) \in \mathbb{R}^{L}$ or $\mathbb{C}^{L}$ of a general nonlinear eigenvalue problem. Depending on the type of application, a wide variety of functions $f$, hence, eigenvalue problems exist. Table 1 shows a few examples of eigenvalue problems frequently encountered in dynamic systems. In general, the eigensolutions depend on the random input $\boldsymbol{X}$ via solution of the matrix characteristic equation

$$
\operatorname{det}\left[f\left(\lambda(\boldsymbol{X}) ; \boldsymbol{A}_{1}(\boldsymbol{X}), \ldots, \boldsymbol{A}_{J}(\boldsymbol{X})\right)\right]=0
$$

and subsequent solution of Eq. (1). A major objective in solving a random eigenvalue problem is to find the probabilistic characteristics of eigenpairs $\left\{\lambda^{(i)}(\boldsymbol{X}), \boldsymbol{\phi}^{(i)}(\boldsymbol{X})\right\}, i=1, \ldots, L$, when the probability law of $\boldsymbol{X}$ is arbitrarily prescribed. Crude Monte Carlo simulation, which can solve any random eigenvalue problem in Table 1 , is computationally inefficient, because it requires solving the matrix characteristic equation for every realization of random matrices $\boldsymbol{A}_{1}(\boldsymbol{X}), \ldots, A_{J}(\boldsymbol{X})$.

\section{Polynomial Dimensional Decomposition}

Let $\lambda(\boldsymbol{X})$, a real-valued, measurable transformation on $(\Omega, \mathcal{F})$, define a relevant eigenvalue of a stochastic dynamic system. In general, the multivariate function $\lambda: \mathbb{R}^{N} \rightarrow \mathbb{R}$ is implicit, is not analytically available, and can only be viewed as a high-dimensional input-output mapping, where the evaluation of the output function $\lambda$ for a given input $\boldsymbol{x}$ requires expensive finite element analysis (FEA). Therefore, methods employed in stochastic analysis must be capable of generating accurate probabilistic characteristics of $\lambda(\boldsymbol{X})$ with an acceptably small number of output function evaluations.

\section{A. Multivariate Function Decomposition}

Consider a continuous, differentiable, real-valued, eigenvalue $\lambda(\boldsymbol{x})$ that depends on $\boldsymbol{x}=\left\{x_{1}, \ldots, x_{N}\right\}^{T} \in \mathbb{R}^{N}$. The dimensional decomposition of $\lambda(\boldsymbol{x})$, also known as analysis of variance [9] or high-dimensional model representation $[10,11]$, represents a finite, hierarchical, convergent expansion of [12]

$$
\begin{aligned}
& \lambda(\boldsymbol{x})=\lambda_{0}+\sum_{i=1}^{N} \lambda_{i}\left(x_{i}\right)+\sum_{i_{1}, i_{2}=1 ; i_{1}<i_{2}}^{N} \lambda_{i_{1} i_{2}}\left(x_{i_{1}}, x_{i_{2}}\right) \\
& \quad+\sum_{i_{1}, i_{2}, i_{3}=1 ; i_{1}<i_{2}<i_{3}}^{N} \lambda_{i_{1} i_{2} i_{3}}\left(x_{i_{1}}, x_{i_{2}}, x_{i_{3}}\right)+\cdots \\
& \quad+\sum_{i_{1}, \cdots, i_{S}=1 ; i_{1}<\cdots<i_{S}}^{N} \lambda_{i_{1} \cdots i_{S}}\left(x_{i_{1}}, \cdots, x_{i_{S}}\right) \cdots+\lambda_{12 \cdots N}\left(x_{1}, \cdots, x_{N}\right)
\end{aligned}
$$

in terms of input variables with increasing dimensions, where $\lambda_{0}$ is a constant, $\lambda_{i}\left(x_{i}\right)$ is a univariate component function representing individual contribution to $\lambda(\boldsymbol{x})$ by input variable $x_{i}$ acting alone, $\lambda_{i_{1} i_{2}}\left(x_{i_{1}}, x_{i_{2}}\right)$ is a bivariate component function describing the cooperative influence of two input variables $\lambda_{i_{1}}$ and $\lambda_{i_{2}}$, $\lambda_{i_{1} i_{2} i_{3}}\left(x_{i_{1}}, x_{i_{2}}, x_{i_{3}}\right)$ is a trivariate component function describing the cooperative influence of three input variables $x_{i_{1}}, x_{i_{2}}$, and $x_{i_{3}}$, $\lambda_{i_{1} \cdots i_{S}}\left(x_{i_{1}}, \ldots, x_{i_{S}}\right)$ is an $S$-variate component function quantifying the cooperative effects of $S$ input variables $x_{i_{1}}, \ldots, x_{i_{S}}$, and so on. (For a complex eigenvalue, $\lambda(\boldsymbol{x})$ represents either the real or the imaginary part.) The last term in Eq. (3) represents any residual dependence of all input variables cooperatively locked together to affect the output function $\lambda$. If

$$
\begin{aligned}
& \tilde{\lambda}_{S}(\boldsymbol{x})=\lambda_{0}+\sum_{i=1}^{N} \lambda_{i}\left(x_{i}\right)+\sum_{i_{1}, i_{2}=1 ; i_{1}<i_{2}}^{N} \lambda_{i_{1} i_{2}}\left(x_{i_{1}}, x_{i_{2}}\right) \\
& +\sum_{i_{1}, i_{2}, i_{3}=1 ; i_{1}<i_{2}<i_{3}}^{N} \lambda_{i_{1} i_{2} i_{3}}\left(x_{i_{1}}, x_{i_{2}}, x_{i_{3}}\right)+\cdots \\
& +\sum_{i_{1}, \ldots, i_{S}=1 ; i_{1}<\cdots<i_{S}}^{N} \lambda_{i_{1} \cdots i_{S}}\left(x_{i_{1}}, \ldots, x_{i_{S}}\right)
\end{aligned}
$$

represents a general $S$-variate approximation of $\lambda(x)$, the univariate $(S=1)$, bivariate $(S=2)$, and trivariate $(S=3)$ approximations, $\tilde{\lambda}_{1}(x), \tilde{\lambda}_{2}(x)$, and $\tilde{\lambda}_{3}(x)$, respectively, provide two-, three-, and fourterm approximants of the finite decomposition in Eq. (3). Similarly, quadrivariate and other higher-variate approximations can be derived by appropriately selecting the value of $S$. The fundamental conjecture underlying this decomposition is that component

Table 1 Random eigenvalue problems in dynamic systems

\begin{tabular}{ll}
\hline \hline Eigenvalue problem $\underline{\text { a }}$ & \multicolumn{1}{c}{ Problem type and application(s) } \\
\hline$[-\lambda(\boldsymbol{X}) \boldsymbol{M}(\boldsymbol{X})+\boldsymbol{K}(\boldsymbol{X})] \phi(\boldsymbol{X})=\mathbf{0}$ & Linear; undamped or proportionally damped systems \\
{$\left[\lambda^{2}(\boldsymbol{X}) \boldsymbol{M}(\boldsymbol{X})+\lambda(\boldsymbol{X}) \boldsymbol{C}(\boldsymbol{X})+\boldsymbol{K}(\boldsymbol{X})\right] \phi(\boldsymbol{X})=\mathbf{0}$} & Quadratic; nonproportionally damped systems; singularity problems \\
{$\left[\lambda(\boldsymbol{X}) \boldsymbol{M}_{1}(\boldsymbol{X})+\boldsymbol{M}_{0}(\boldsymbol{X})+\boldsymbol{M}_{1}^{T}(\boldsymbol{X}) / \lambda(\boldsymbol{X})\right] \phi(\boldsymbol{X})=\mathbf{0}$} & Palindromic; acoustic emissions in high-speed trains \\
{$\left[\sum_{k} \lambda^{k}(\boldsymbol{X}) \boldsymbol{A}_{k}(\boldsymbol{X})\right] \phi(\boldsymbol{X})=\mathbf{0}$} & Polynomial; control and dynamics problems \\
{$\left[\lambda(\boldsymbol{X}) \boldsymbol{M}(\boldsymbol{X})-\boldsymbol{K}(\boldsymbol{X})+\sum_{k} \frac{\lambda^{q}(\boldsymbol{X}) \boldsymbol{C}_{k}(\boldsymbol{X})}{a_{k}-\lambda(\boldsymbol{X})}\right] \phi(\boldsymbol{X})=\mathbf{0}$} & Rational; plate vibration $(q=1)$, fluid-structure vibration $(q=2)$, vibration of viscoelastic materials \\
\hline \hline
\end{tabular}

${ }^{\mathrm{a}} \boldsymbol{M}(\boldsymbol{X}), \boldsymbol{C}(\boldsymbol{X})$, and $\boldsymbol{K}(\boldsymbol{X})$ are the mass, damping, stiffness matrices, respectively; $\boldsymbol{M}_{0}(X), \boldsymbol{M}_{1}(X), \boldsymbol{A}_{k}(X)$, and $\boldsymbol{C}_{k}(X)$ are various coefficient matrices. 
functions arising in the function decomposition will exhibit insignificant $S$-variate effects cooperatively when $S \rightarrow N$, leading to useful lower-variate approximations of $\lambda(\boldsymbol{x})$. When $S=N, \tilde{\lambda}_{S}(\boldsymbol{x})$ converges to the exact function $\lambda(x)$. In other words, Eq. (4) generates a hierarchical and convergent sequence of approximations of $\lambda(\boldsymbol{x})$. Theoretical details of this decomposition are available elsewhere [9-13].

\section{B. Fourier-Polynomial Expansion}

Let $\mathcal{L}_{2}\left(\Omega_{i}, \mathcal{F}_{i}, P_{i}\right)$ be a Hilbert space that is equipped with a set of complete orthonormal bases $\left\{\psi_{i j}\left(x_{i}\right) ; j=0,1, \cdots\right\}$, which is consistent with the probability measure of $X_{i}$. For example, classical orthonormal polynomials, including Hermite, Legendre, and Jacobi polynomials, can be used when $X_{i}$ follows Gaussian, uniform, and Beta probability distributions, respectively [14]. Defined on the product probability triple $\left(\times_{k=1}^{k=S} \Omega_{i_{k}}, \times_{k=1}^{k=S} \mathcal{F}_{i_{k}}, \times_{k=1}^{k=S} P_{i_{k}}\right)$, denote the space of square integrable $S$-variate component functions of $\lambda$ by

$$
\begin{aligned}
\mathcal{L}_{2} & \left(\times_{k=1}^{k=S} \Omega_{i_{k}}, \times_{k=1}^{k=S} \mathcal{F}_{i_{k}}, \times_{k=1}^{k=S} P_{i_{k}}\right) \\
& :=\left\{\lambda_{i_{1} \cdots i_{S}}\left(X_{i_{1}}, \ldots, X_{i_{S}}\right): \int_{\mathbb{R}^{S}} \lambda_{i_{1} \cdots i_{S}}^{2}\left(x_{i_{1}}, \ldots, x_{i_{S}}\right)\right. \\
& \left.\times \prod_{k=1}^{S} f_{i_{k}}\left(x_{i_{k}}\right) \mathrm{d} x_{i_{k}}<\infty\right\}
\end{aligned}
$$

which is also a Hilbert space. Because the joint probability density of $\left\{X_{i_{1}}, \ldots, X_{i_{S}}\right\}^{T}$ is separable (independence), the tensor product

$$
\left\{\prod_{k=1}^{S} \psi_{i_{k} j_{k}}\left(x_{i_{k}}\right)\right\}
$$

constitutes an orthonormal polynomial basis in $\mathcal{L}_{2}\left(\times_{k=1}^{k=S} \Omega_{i_{k}}\right.$, $\left.\times_{k=1}^{k=S} \mathcal{F}_{i_{k}}, \times_{k=1}^{k=S} P_{i_{k}}\right)$. Therefore, there exists a Fourier-polynomial expansion

$$
\lambda_{i_{1} \cdots i_{S}}\left(x_{i_{1}}, \ldots, x_{i_{S}}\right)=\sum_{j_{S}=1}^{\infty} \ldots \sum_{j_{1}=1}^{\infty} C_{i_{1} \cdots i_{S} j_{1} \cdots j_{S}} \prod_{k=1}^{S} \psi_{i_{k} j_{k}}\left(x_{i_{k}}\right)
$$

with

$$
C_{i_{1} \cdots i_{S} j_{1} \cdots j_{S}}=\int_{\mathbb{R}^{S}} \lambda_{i_{1} \cdots i_{S}}\left(x_{i_{1}}, \ldots, x_{i_{S}}\right) \prod_{k=1}^{S} \psi_{i_{k} j_{k}}\left(x_{i_{k}}\right) f_{i_{k}}\left(x_{i_{k}}\right) \mathrm{d} x_{i_{k}}
$$

representing the expansion coefficient for the $S$-variate component function. By minimizing an error functional associated with a given $\lambda(\boldsymbol{x})$ and the joint probability density of $\left\{X_{i_{1}}, \ldots, X_{i_{S}}\right\}^{T}$, the coefficients $\lambda_{0}$ and $C_{i_{1} \cdots i_{S} j_{1} \cdots j_{S}}$ can be expressed by $N$-dimensional integrals [1ㅡㄹ

$$
\lambda_{0}=\int_{\mathbb{R}^{N}} \lambda(\boldsymbol{x}) f_{X}(\boldsymbol{x}) \mathrm{d} \boldsymbol{x}
$$

and

$$
C_{i_{1} \cdots i_{S} j_{1} \cdots j_{S}}=\int_{\mathbb{R}^{N}} \lambda(\boldsymbol{x}) \prod_{k=1}^{S} \psi_{i_{k} j_{k}}\left(x_{i_{k}}\right) f_{\boldsymbol{X}}(\boldsymbol{x}) \mathrm{d} \boldsymbol{x}
$$

Because the right side of Eq. (ㅁ) is an infinite series, it must be truncated, say, by $m$ terms in each variable, yielding a Fourierpolynomial approximation

$$
\lambda_{i_{1} \cdots i_{S}}\left(x_{i_{1}}, \ldots, x_{i_{S}}\right) \cong \sum_{j_{S}=1}^{m} \cdots \sum_{j_{1}=1}^{m} C_{i_{1} \cdots i_{S} j_{1} \cdots j_{S}} \prod_{k=1}^{S} \psi_{i_{k} j_{k}}\left(x_{i_{k}}\right)
$$

which approaches $\lambda_{i_{1} \cdots i_{S}}\left(x_{i_{1}}, \ldots, x_{i_{S}}\right)$ in Eq. ( $\left.\underline{6}\right)$ in the mean square sense as $m \rightarrow \infty$.

The Fourier-polynomial approximation is valid for any finitedimensional Hilbert space $\mathcal{L}_{2}\left(\times_{k=1}^{k=S} \Omega_{i_{k}}, \times_{k=1}^{k=S} \mathcal{F}_{i_{k}}, \times_{k=1}^{k=S} P_{i_{k}}\right)$ with $1 \leq S \leq N$. In other words, Eq. (10) can represent all component functions of the multivariate function decomposition in Eq. (3). In particular, when $S=1,2$, and 3, Eq. (10) reduces to

$$
\begin{gathered}
\lambda_{i}\left(x_{i}\right) \cong \sum_{j=1}^{m} \alpha_{i j} \psi_{i j}\left(x_{i}\right) \\
\lambda_{i_{1} i_{2}}\left(x_{i_{1}}, x_{i_{2}}\right) \cong \sum_{j_{2}=1}^{m} \sum_{j_{1}=1}^{m} \beta_{i_{1} i_{2} j_{1} j_{2}} \psi_{i_{1} j_{1}}\left(x_{i_{1}}\right) \psi_{i_{2} j_{2}}\left(x_{i_{2}}\right)
\end{gathered}
$$

and

$$
\begin{aligned}
& \lambda_{i_{1} i_{2} i_{3}}\left(x_{i_{1}}, x_{i_{2}}, x_{i_{3}}\right) \\
& \cong \sum_{j_{3}=1}^{m} \sum_{j_{2}=1}^{m} \sum_{j_{1}=1}^{m} \gamma_{i_{1} i_{2} i_{3} j_{1} j_{2} j_{3}} \psi_{i_{1} j_{1}}\left(x_{i_{1}}\right) \psi_{i_{2} j_{2}}\left(x_{i_{2}}\right) \psi_{i_{3} j_{3}}\left(x_{i_{3}}\right)
\end{aligned}
$$

where

$$
\begin{gathered}
\alpha_{i j}:=\int_{\mathbb{R}^{N}} \lambda(\boldsymbol{x}) \psi_{i j}\left(x_{i}\right) f_{\boldsymbol{X}}(\boldsymbol{x}) \mathrm{d} \boldsymbol{x} \\
\beta_{i_{1} i_{2} j_{1} j_{2}}:=\int_{\mathbb{R}^{N}} \lambda(\boldsymbol{x}) \psi_{i_{1} j_{1}}\left(x_{i_{1}}\right) \psi_{i_{2} j_{2}}\left(x_{i_{2}}\right) f_{\boldsymbol{X}}(\boldsymbol{x}) \mathrm{d} \boldsymbol{x}
\end{gathered}
$$

and

$$
\gamma_{i_{1} i_{2} i_{3} j_{1} j_{2} j_{3}}:=\int_{\mathbb{R}^{N}} \lambda(\boldsymbol{x}) \psi_{i_{1} j_{1}}\left(x_{i_{1}}\right) \psi_{i_{2} j_{2}}\left(x_{i_{2}}\right) \psi_{i_{3} j_{3}}\left(x_{i_{3}}\right) f_{\boldsymbol{X}}(\boldsymbol{x}) \mathrm{d} \boldsymbol{x}
$$

are the corresponding expansion coefficients. Applying Eqs. (10-16) to an $S$-variate approximation of Eq. (3) yields

$$
\begin{aligned}
& \tilde{\lambda}_{S}(\boldsymbol{X}) \cong \lambda_{0}+\sum_{i=1}^{N} \sum_{j=1}^{m} \alpha_{i j} \psi_{i j}\left(X_{i}\right) \\
& +\sum_{i_{1}, i_{2}=1 ; i_{1}<i_{2}}^{N} \sum_{j_{2}=1}^{m} \sum_{j_{1}=1}^{m} \beta_{i_{1} i_{2} j_{1} j_{2}} \psi_{i_{1} j_{1}}\left(X_{i_{1}}\right) \psi_{i_{2} j_{2}}\left(X_{i_{2}}\right)+\cdots \\
& +\sum_{i_{1}, \cdots, i_{S}=1 ; i_{1}<\cdots<i_{S}}^{N} \sum_{j_{S}=1}^{m} \cdots \sum_{j_{1}=1}^{m} C_{i_{1} \cdots i_{S} j_{1} \cdots j_{S}} \prod_{k=1}^{S} \psi_{i_{k} j_{k}}\left(X_{i_{k}}\right)
\end{aligned}
$$

which, for $S=N$, converges to $\lambda(\boldsymbol{X})$ in the mean square sense as $m \rightarrow \infty$. Once the embedded coefficients $\lambda_{0}, \alpha_{i j}, \beta_{i_{1} i_{2} j_{1} j_{2}}$, and $C_{i_{1} \cdots i_{S} j_{1} \cdots j_{S}}$ are calculated, as described in a forthcoming section, Eq. (17) furnishes an approximate but explicit map $\tilde{\lambda}_{S}: \mathbb{R}^{N} \rightarrow \mathbb{R}$ that can be viewed as a surrogate of the exact map $\lambda: \mathbb{R}^{N} \rightarrow \mathbb{R}$, which describes the input-output relation from a complicated numerical simulation. Therefore, any probabilistic characteristic of $\lambda(X)$, including its statistical moments and probability distribution, can be easily estimated by performing a Monte Carlo simulation of $\tilde{\lambda}_{S}(\boldsymbol{X})$ rather than of $\lambda(\boldsymbol{X})$. The simulation of $\tilde{\lambda}_{S}(\boldsymbol{X})$, which entails evaluation of simple analytical functions, can be performed for an arbitrarily large sample size. In contrast, the simulation of $\lambda(\boldsymbol{X})$, referred to as crude Monte Carlo simulation in this paper, requires expensive numerical calculations and can therefore be prohibitive when estimating tail probabilities.

\section{Dimension-Reduction Integration for Calculating Expansion Coefficients}

The determination of the expansion coefficients, which involve $N$-dimensional integrals over $\mathbb{R}^{N}$, is computationally prohibitive when $N$ is large. Instead, a dimension-reduction integration, presented as follows, can be applied to estimate the coefficients efficiently.

Let $\boldsymbol{c}=\left\{c_{1}, \ldots, c_{N}\right\}^{T}$ be the mean value of $\boldsymbol{X}$ and $\lambda\left(c_{1}, \ldots\right.$, $\left.c_{k_{1}-1}, x_{k_{1}}, c_{k_{1}+1}, \ldots, c_{k_{R-k}-1}, x_{k_{R-k}}, c_{k_{R-k}+1}, \ldots, c_{N}\right)$ represent an $(R-k)$ th dimensional component function of $\lambda(\boldsymbol{x})$, where $S \leq R \leq$ 
$N$ and $k=0, \ldots, R$. For example, when $R=1$, the zerodimensional component function, which is constant, is $\lambda(\boldsymbol{c})$ and the one-dimensional component functions are $\lambda\left(x_{1}, c_{2}, \ldots, c_{N}\right)$, $\lambda\left(c_{1}, x_{2}, \ldots, c_{N}\right), \ldots, \lambda\left(c_{1}, c_{2}, \ldots, x_{N}\right)$. Using $\mathrm{Xu}$ and Rahman's [13] multivariate function theorem, it can be shown that a special $R$ variate approximation of $\lambda(\boldsymbol{x})$, defined by

$$
\begin{aligned}
& \hat{\lambda}_{R}(\boldsymbol{x}):=\sum_{k=0}^{R}(-1)^{k}\left(\begin{array}{c}
N-R+k-1 \\
k
\end{array}\right) \times \sum_{k_{1}, \ldots, k_{R-k}=1 ; k_{1}<\cdots<k_{R-k}}^{N} \\
& \quad \times \lambda\left(c_{1}, \ldots, c_{k_{1}-1}, x_{k_{1}}, c_{k_{1}+1}, \ldots, c_{k_{R-k}-1}, x_{k_{R-k}}, c_{k_{R-k}+1}, \ldots, c_{N}\right)
\end{aligned}
$$

consists of all terms of the Taylor series of $\lambda(x)$ that have less than or equal to $R$ variables. The expanded form of Eq. (18), when compared with the Taylor expansion of $\lambda(\boldsymbol{x})$, reveals that the residual error in $\hat{\lambda}_{R}(x)$ includes terms of dimensions $R+1$ and higher. All higherorder $R$ - and lower-variate terms of $\lambda(x)$ are included in Eq. (18), which should therefore generally provide a higher-order approximation of a multivariate function than equations derived from first- or second-order Taylor expansions. Therefore, for $R<N$, an $N$ dimensional integral can be efficiently estimated by at most $R$ dimensional integrations, if the contributions from terms of dimensions $R+1$ and higher are negligible.

Substituting $\lambda(\boldsymbol{x})$ in Eqs. ( $\underline{8})$ and (9) by $\hat{\lambda}_{R}(\boldsymbol{x})$, the coefficients can be estimated from

$$
\begin{aligned}
\lambda_{0} & \cong \sum_{k=0}^{R}(-1)^{k}\left(\begin{array}{c}
N-R+k-1 \\
k
\end{array}\right)_{k_{1}, \cdots, k_{R-k}=1 ; k_{1}<\cdots<k_{R-k}} \sum^{N} \\
& \times \int_{\mathbb{R}^{R-k}} \lambda\left(c_{1}, \ldots, c_{k_{1}-1}, x_{k_{1}}, c_{k_{1}+1}, \ldots, c_{k_{R-k}-1}, x_{k_{R-k}}, c_{k_{R-k}+1}, \ldots, c_{N}\right) \\
& \times \prod_{s=1}^{R-k} f_{k_{s}}\left(x_{k_{s}}\right) \mathrm{d} x_{k_{s}}
\end{aligned}
$$

and

$$
\begin{aligned}
& C_{i_{1} \cdots i_{S} j_{1} \cdots j_{S}} \cong \sum_{k=0}^{R}(-1)^{k}\left(\begin{array}{c}
N-R+k-1 \\
k
\end{array}\right)_{k_{1}, \ldots, k_{R-k}=1 ; k_{1}<\cdots<k_{R-k}} \sum_{\mathbb{R}^{R-k}}^{N} \lambda\left(c_{1}, \ldots, c_{k_{1}-1}, x_{k_{1}}, c_{k_{1}+1}, \ldots, c_{k_{R-k}-1}, x_{k_{R-k}}, c_{k_{R-k}+1}, \ldots, c_{N}\right) \\
& \times \int_{s=1}^{S} \psi_{i_{s} j_{s}}\left(x_{i_{s}}\right) \prod_{s=1}^{R-k} f_{k_{s}}\left(x_{k_{s}}\right) \mathrm{d} x_{k_{s}}
\end{aligned}
$$

which require evaluating at most $R$-dimensional integrals. The proposed equations, Eqs. (19) and (20), are substantially simpler and more efficient than performing one $N$-dimensional integration, as in Eqs. (8) and (9), particularly when $R \ll N$. Hence, the computational effort in calculating the coefficients is significantly lowered using the dimension-reduction integration. When $R=1,2$, or 3, Eqs. (19) and (20) involve one-, at most two-, and at most three-dimensional integrations, respectively. Nonetheless, numerical integration is still required for a general function $\lambda$. The integration nodes and associated weights, which depend on the probability distribution of $X_{i}$, can be obtained from Gaussian quadrature rules. In performing the dimension-reduction integration, the value of $R$ should be selected in such a way that it is either equal to or greater than the value of $S$, which defines the truncation of Eq. (3). Then, all expansion coefficients of $S$ - or lower-variate approximations of $\lambda(\boldsymbol{x})$ will have nontrivial solutions [12].

\section{Computational Effort}

The $S$-variate approximation in the polynomial decomposition method requires evaluation of the deterministic coefficients $\lambda_{0}$ and $C_{i_{1} \cdots i_{S} j_{1} \cdots j_{S}}$. If these coefficients are estimated by at most $R$ dimensional $(R \geq S \geq 1)$ numerical integration with an $n$-point quadrature rule in Eqs. (19) and (20), the following deterministic responses (eigenvalue evaluations) are required: $\lambda(\boldsymbol{c}), \lambda\left(c_{1}, \ldots\right.$, $\left.c_{k_{1}-1}, x_{k_{1}}^{\left(j_{1}\right)}, c_{k_{1}+1}, \ldots, c_{k_{R}-1}, x_{k_{R}}^{\left(j_{R}\right)}, c_{k_{R}+1}, \ldots, c_{N}\right)$ for $k_{1}, \ldots, k_{R}=$ $1, \ldots, N$ and $j_{1}, \ldots, j_{R}=1, \ldots, n$, where the superscripts to the variables indicate corresponding integration points. Therefore, the total cost for an $S$-variate polynomial dimensional decomposition entails a maximum of

$$
\sum_{k=0}^{k=R}\left(\begin{array}{c}
N \\
R-k
\end{array}\right) n^{R-k}
$$

eigenvalue evaluations. For example, the univariate $(S=R=1)$, bivariate $(S=R=2)$, and trivariate $(S=R=3)$ approximations require $n N+1$ (linear), $N(N-1) n^{2} / 2+n N+1$ (quadratic), and $N(N-1)(N-2) n^{3} / 6+N(N-1) n^{2} / 2+n N+1$ (cubic) eigenvalue evaluations, respectively. If the integration points include a common point in each coordinate $x_{i}$, as in the forthcoming example section, then the numbers of eigenvalue evaluations reduce to

$$
\sum_{k=0}^{k=R}\left(\begin{array}{c}
N \\
R-k
\end{array}\right)(n-1)^{R-k}
$$

Consequently, the univariate $(S=R=1)$, bivariate $(S=R=2)$, and trivariate $(S=R=3)$ approximations require $(n-1) N+1$, $N(N-1)(n-1)^{2} / 2+(n-1) N+1$, and $N(N-1)(N-2)(n-$ $1)^{3} / 6+N(N-1)(n-1)^{2} / 2+(n-1) N+1$ eigenvalue evaluations, respectively.

Because the decomposition in Eq. (17) is structured with respect to the degree of cooperativity between a finite number of random variables, the exponential complexity associated with the curse of dimensionality has been reduced to a polynomic complexity with respect to $N$ or $n$. If a response is highly nonlinear, but contains rapidly diminishing cooperative effects of multiple random variables, the polynomial dimensional decomposition is effective. This is because the lower-variate (univariate, bivariate, etc.) terms of the decomposition can be nonlinear by selecting appropriate orders of orthogonal polynomials.

\section{Numerical Examples}

Three numerical examples demonstrating the polynomial dimensional decomposition method for obtaining the probability distributions of natural frequencies or eigenvalues are presented. Comparisons have been made with select existing methods and crude Monte Carlo simulation to evaluate the accuracy and efficiency of the decomposition method. For the first two examples, the eigenvalues were calculated by a hybrid double-shifted LR-QR algorithm [15]. A Lanczos algorithm embedded in the commercial code AB $\overline{A Q} U S$ (version 6.8) [16] was employed for the third example. For the non-Gaussian random input, all original random variables were transformed into standard Gaussian random variables, employing Hermite orthonormal polynomials as bases and the Gauss-Hermite quadrature rule for calculating the expansion coefficients. The expansion coefficients were estimated by dimension-reduction integration with $R=S$, so that an $S$-variate decomposition method requires at most $S$-variate numerical integration. In examples 1 and 2, the sample sizes for crude Monte Carlo simulation and the embedded Monte Carlo simulation of the decomposition method are both $10^{6}$. The respective sample sizes are 5000 and $10^{6}$ in example 3 . The polynomial order $m$ varies depending on the example, but in all cases the number of integration points $n=m+1$.

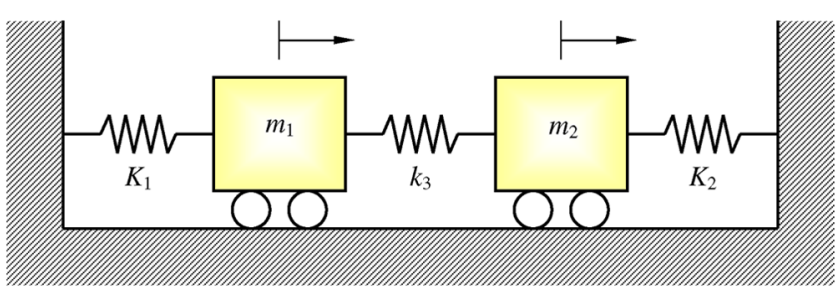

Fig. 1 A 2-degree-of-freedom, undamped, spring-mass system. 


\section{A. Example 1: Undamped, Spring-Mass System}

Consider a 2-degree-of-freedom, undamped, spring-mass system, shown in Fig. 1, with deterministic mass and random stiffness matrices

$$
\begin{aligned}
& \boldsymbol{m}=\left[\begin{array}{cc}
m_{1} & 0 \\
0 & m_{2}
\end{array}\right] \in \mathbb{R}^{2 \times 2} \text { and } \\
& \boldsymbol{K}(\boldsymbol{X})=\left[\begin{array}{cc}
K_{1}(\boldsymbol{X})+k_{3} & -k_{3} \\
-k_{3} & K_{2}(\boldsymbol{X})+k_{3}
\end{array}\right] \in \mathbb{R}^{2 \times 2}
\end{aligned}
$$

respectively, where $m_{1}=1 \mathrm{~kg}, m_{2}=1.5 \mathrm{~kg}, K_{1}(\boldsymbol{X})=1000(1+$ $\left.0.25 X_{1}\right) \mathrm{N} / \mathrm{m}, \quad K_{2}(X)=1100\left(1+0.25 X_{2}\right) \mathrm{N} / \mathrm{m}, \quad$ and $\quad k_{3}=$ $100 \mathrm{~N} / \mathrm{m}$ [5]. The random variables $X_{1}$ and $X_{2}$ are independent
$(N=2)$, each following standard Gaussian distribution with zero mean and unit variance [5]. From the size of the matrices, there exist two real-valued random eigenvalues, $\lambda_{1}(X)$ and $\lambda_{2}(X)$, which are sorted into an ascending order.

Because the eigenvalues are nonpolynomial functions of input, a convergence study with respect to $S$ and $m$ is required to calculate their probability distributions accurately. Figure 2 a presents the marginal probability distributions of $\lambda_{1}(\boldsymbol{X})$ and $\bar{\lambda}_{2}(\boldsymbol{X})$ by the univariate $(S=1)$ and bivariate $(S=2)$ polynomial decomposition methods for several values of $m$. The figure also includes corresponding distributions generated by crude Monte Carlo simulation, providing benchmark solutions. The tail probabilities from both versions of the decomposition method converge when $m$ reaches 5 or 6 , but the Monte Carlo solutions at low probabilities are
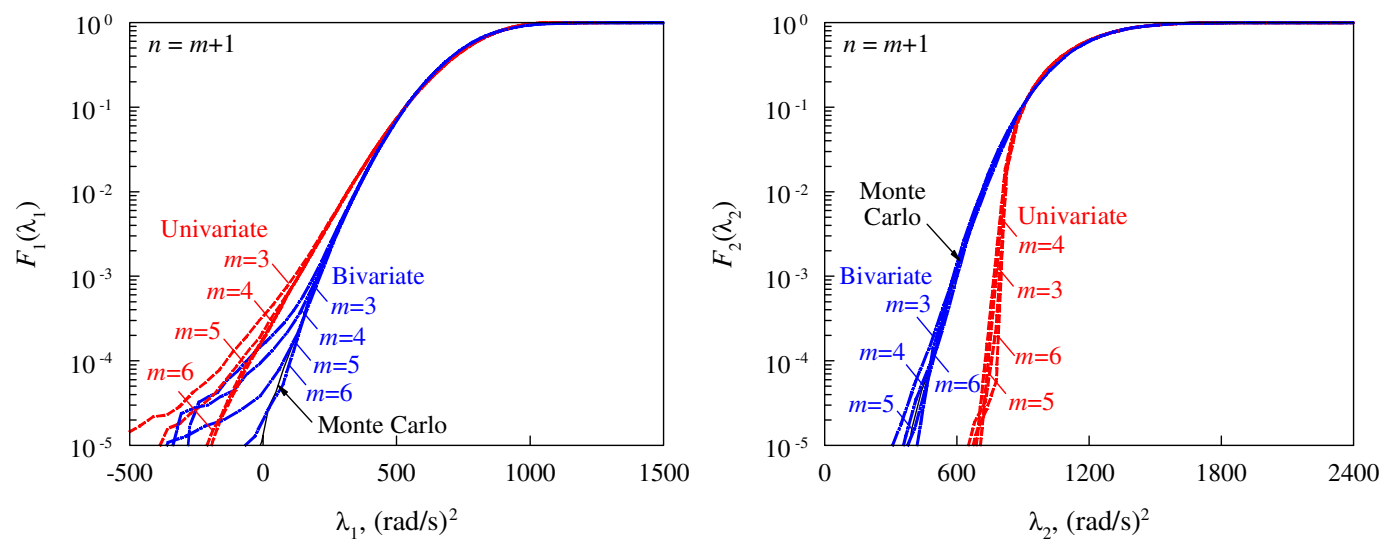

a)
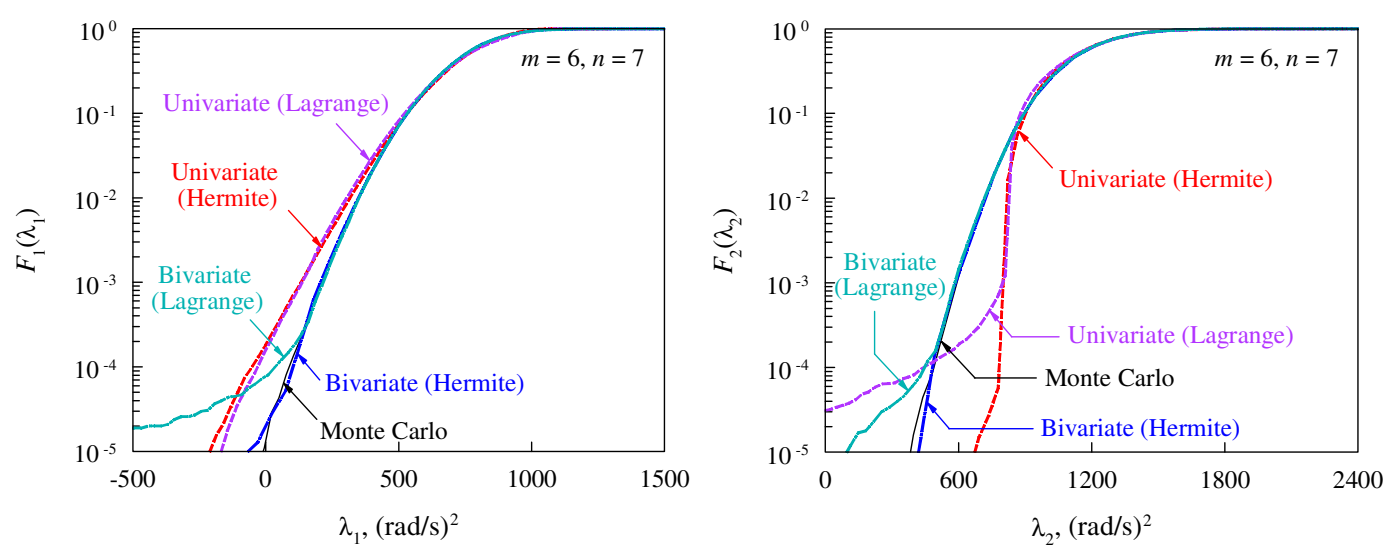

b)
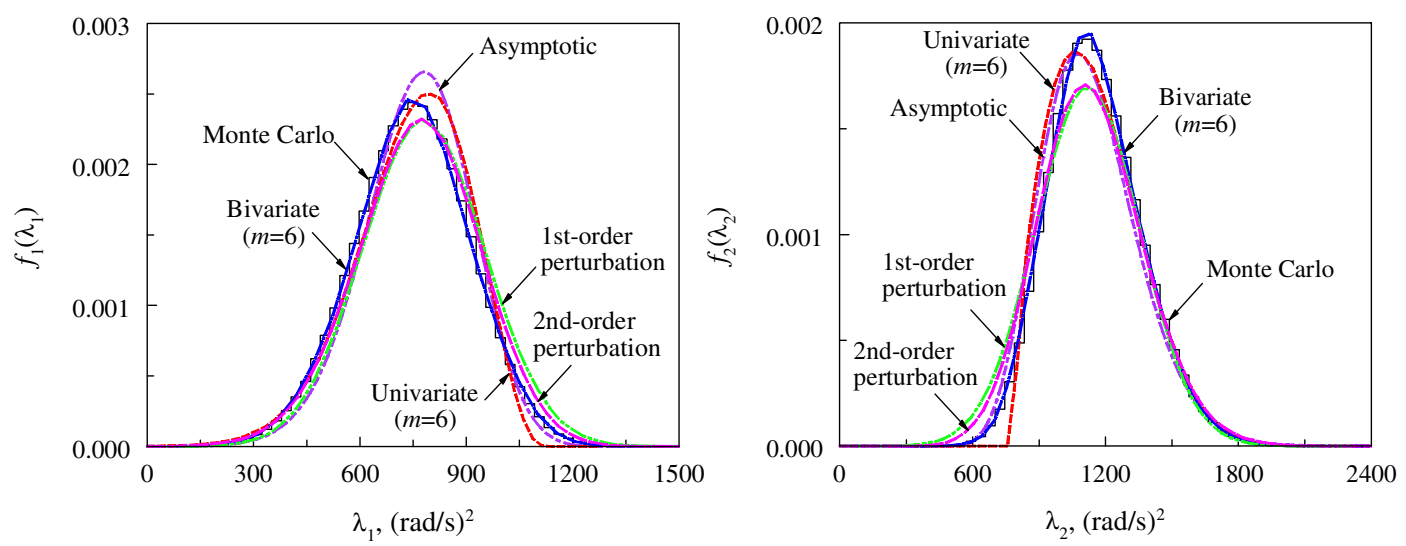

c)

Fig. 2 Eigenvalues of a 2-degree-of-freedom system: a) convergence of probability distributions by polynomial decomposition methods, b) probability distributions by existing and proposed decomposition methods, and c) probability densities by various methods. 

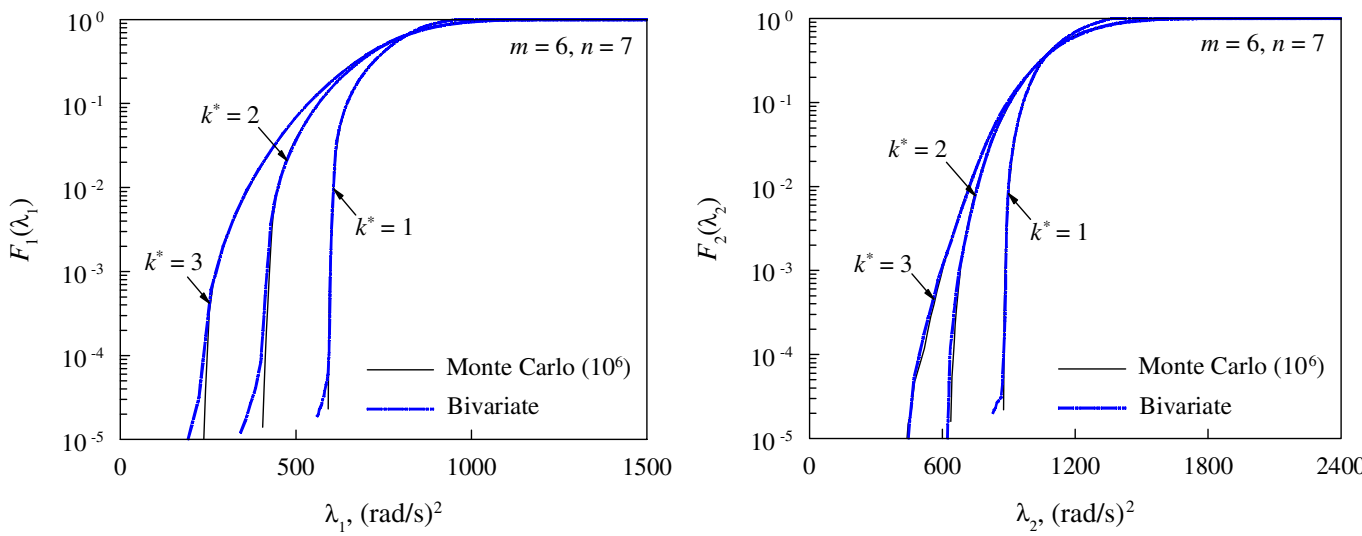

a)
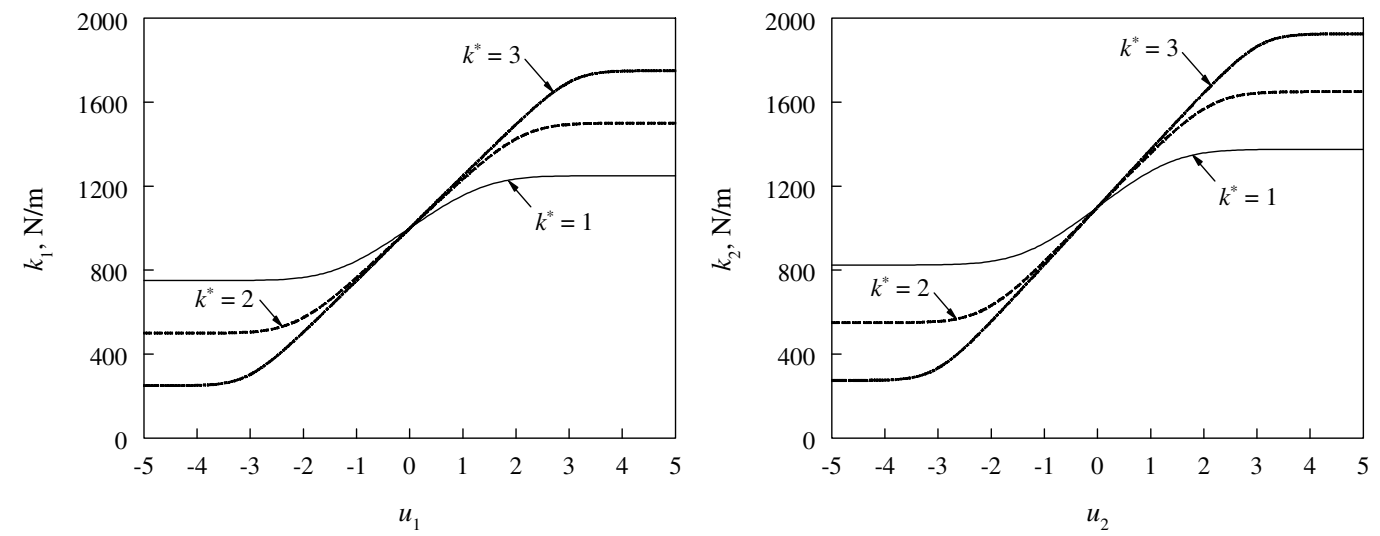

b)

Fig. 3 Eigenvalues for truncated random stiffnesses: a) tail probability distributions, and b) transformations between truncated Gaussian and standard Gaussian variables.

better predicted by the bivariate method than the univariate method. This is primarily due to the absence of cooperative terms in the univariate approximation. The results from an existing decomposition method employing sample points and a sixth-order Lagrange interpolation [7], shown in Fig. 2b, reveal the same trend, except at the tail end, where the probability distributions are less accurate. The accuracy should increase by raising the order of Lagrange interpolations, but such improvements will require more computational effort. In contrast, the proposed decomposition method yields more accurate tail probabilities than the existing decomposition method for the same order and, more important, does not require sample points. It is worth noting that the orders of polynomials required for calculating tail probabilities depend on the computed probability and are usually larger than those required for calculating moments [7].

Because $\boldsymbol{X}=\left\{X_{1}, X_{2}\right\}^{T} \in \mathbb{R}^{2}$ is Gaussian, the stiffness matrix $\boldsymbol{K}(\boldsymbol{X})$ may become negative definite. Therefore, there exists a finite probability, regardless how small, that the resultant eigenvalues may be negative. Indeed, the results of Fig. 2a (left panel) confirm this assertion, yielding negative samples of the first eigenvalue by all three methods, although the probability of such an occurrence should diminish with lower standard deviations of $K_{i}$. Because of the larger mean, no negative second eigenvalues were detected, at least for probabilities greater than $10^{-5}$. A Gaussian description of the random input is unrealistic, but it was still selected in this example to compare the results with those from the existing methods that require or employ the Gaussian assumption $[\underline{5}, 6]$.

Figure $2 \mathrm{c}$ plots the marginal probability densities or histograms of $\lambda_{1}(\boldsymbol{X})$ and $\lambda_{2}(\boldsymbol{X})$ calculated by a number of methods, including the univariate $(S=1, m=6)$ and bivariate $(S=2, m=6)$ polynomial decomposition methods, first- and second-order perturbation methods, the asymptotic method, and crude Monte Carlo simulation. Compared with the Monte Carlo result, the proposed decomposition

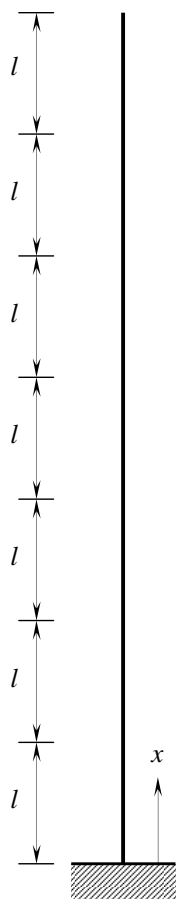

a) of-freedom discrete model.

b)

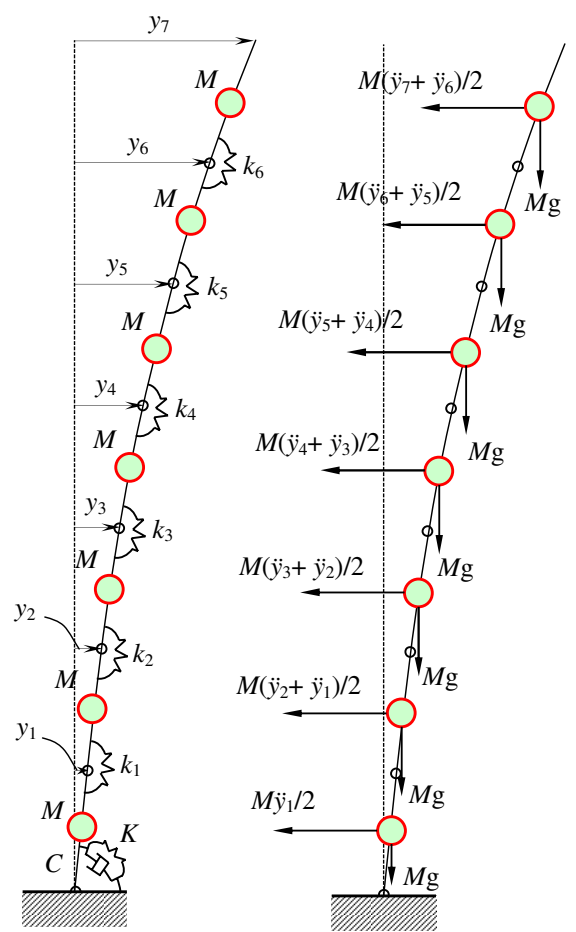


methods, especially the bivariate version, provide tail characteristics of both eigenvalues more accurately than other approximate methods examined.

For a more realistic input, consider truncated Gaussian distributions of random stiffnesses $K_{i}$, expressed by $\left[\Phi\left(k_{i}-\mu_{i}\right) /\right.$ $\left.\left.\left.\left.\left.\sigma_{i}\right)-\Phi\left(a_{i}-\mu_{i}\right) / \sigma_{i}\right)\right] /\left[\Phi\left(b_{i}-\mu_{i}\right) / \sigma_{i}\right)-\Phi\left(a_{i}-\mu_{i}\right) / \sigma_{i}\right)\right], \quad i=1$, 2 , where $\Phi(\cdot)$ is the cumulative distribution function of a standard Gaussian variable, and $\mu_{1}=1000 \mathrm{~N} / \mathrm{m}, \mu_{2}=1100 \mathrm{~N} / \mathrm{m}, \sigma_{1}=$ $250 \mathrm{~N} / \mathrm{m}, \sigma_{2}=275 \mathrm{~N} / \mathrm{m}$, and $a_{i}$ and $b_{i}$ are the lower and upper bounds, respectively, of $K_{i}$. Let the standardized bounds be $\left.\left.-\left(a_{i}-\mu_{i}\right) / \sigma_{i}\right)=\left(b_{i}-\mu_{i}\right) / \sigma_{i}\right)=k^{*}$, where $k^{*} \geq 0$ is a truncation parameter, defining the support $\left[-\left(\mu_{i}+k^{*} \sigma_{i}\right),+\left(\mu_{i}+k^{*} \sigma_{i}\right)\right]$ of the probability density function of $K_{i}$. By transforming truncated Gaussian to standard Gaussian variables and then employing Fourier-Hermite expansions, the tail probability distributions of the resultant eigenvalues were calculated by a sixth-order bivariate polynomial decomposition and crude Monte Carlo simulation $\left(10^{6}\right.$ samples) for several values of $k^{*}: 1,2$, and 3 . The comparative results in Fig. $\underline{3 a}$ continue to demonstrate the high accuracy of the decomposition method for all three values of $k^{*}$. As long as the transformation between $K_{i}$ and its standard Gaussian image $U_{i}$ does not induce overly large nonlinearity, as is the case in this problem and plotted in Fig. 3 b, the proposed method should also be applicable to non-Gaussian random input.

\section{B. Example 2: Freestanding Beam}

The second example involves the free vibration of a tall, freestanding beam, shown in Fig. 4a [8]. Figure $\underline{4 b}$ represents a lumpedparameter model of the beam, which comprises seven rigid, massless
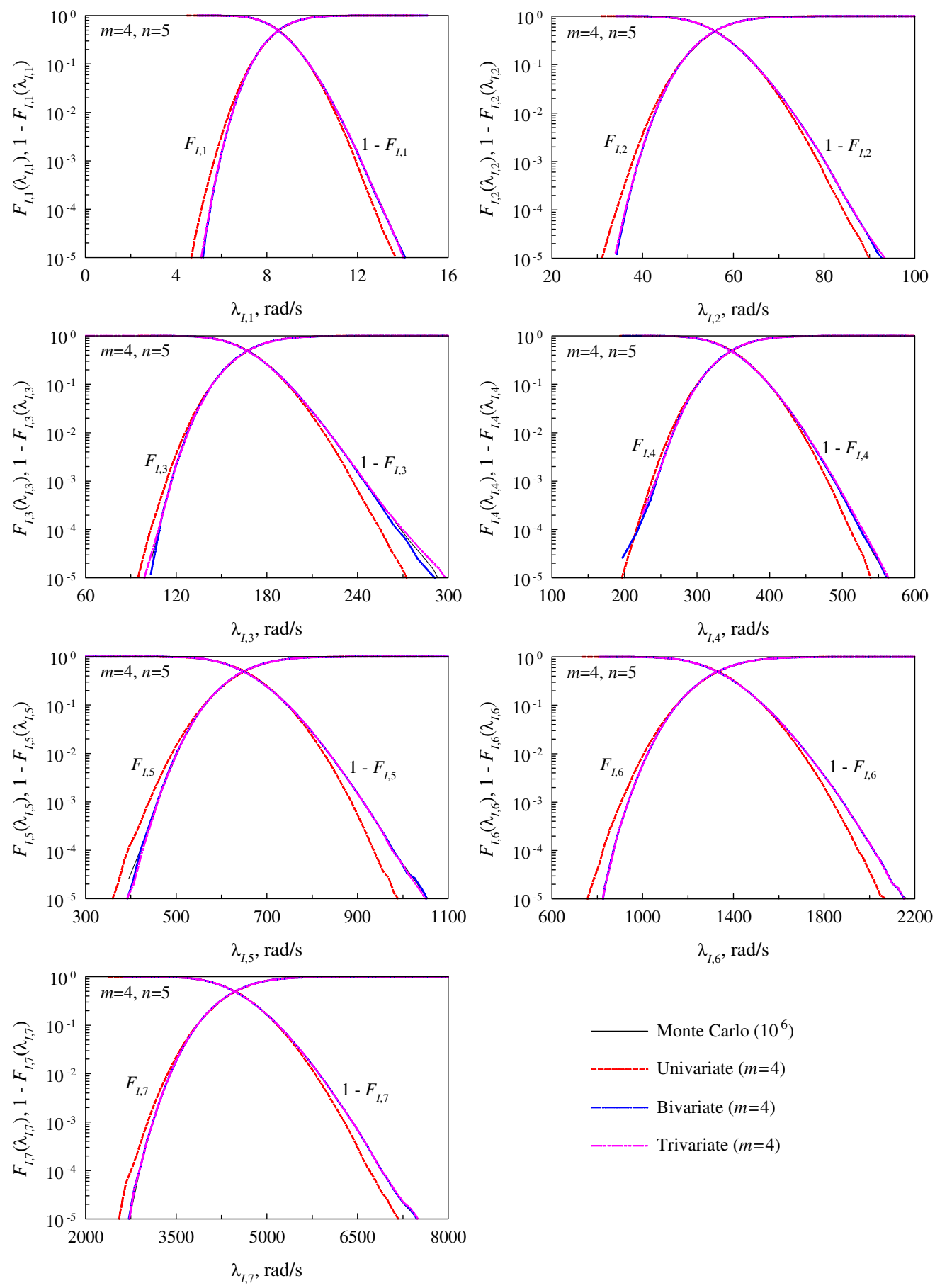

Fig. 5 Tail probability distributions of the imaginary parts of eigenvalues of a beam. 
links hinged together. The mass of the beam is represented by seven random point masses located at the center of each link. No damping was assumed except at the bottom joint, where the random, rotational, viscous damping coefficient due to the foundation pad is $C$. The random rotational stiffness at the bottom of the beam, controlled by the lower half of the bottom link and the flexibility of the foundation pad, is $K$. The independent random variables $M, C$, and $K$ are lognormally distributed with respective means $3000 \mathrm{~kg}, 2 \times 10^{7} \mathrm{~N} \cdot \mathrm{m} \cdot \mathrm{s} / \mathrm{rad}$, and $2 \times 10^{9} \mathrm{~N} \cdot \mathrm{m} / \mathrm{rad}$ and have a $20 \%$ coefficient of variation. The flexural rigidity of the beam is represented by six rotational springs between links with stiffnesses $k(x)=k\left(x_{i}\right), i=1, \ldots, 6$, where $x_{i}=i l i=1, \ldots, 6$, and $l=6 \mathrm{~m}$. The spatially varying spring stiffness $k(x)=c_{\alpha} \exp [\alpha(x)]$ is an independent, homogeneous, lognormal random field with mean $\mu_{k}=2 \times 10^{9} \mathrm{~N} \cdot \mathrm{m} / \mathrm{rad}$ and coefficient of variation $v_{k}=0.2$, where $c_{\alpha}=\mu_{k} / \sqrt{1+v_{k}^{2}}$ and $\alpha(x)$ is a zero-mean, homogeneous, Gaussian random field with variance $\sigma_{\alpha}^{2}=\ln \left(1+v_{k}^{2}\right)$ and covariance function $\Gamma_{\alpha}(u):=\mathbb{E}[\alpha(x) \alpha(x+u)]=\sigma_{\alpha}^{2} \exp (-|u| / l)$. A discretization of $\alpha(x)$ yields the zero-mean Gaussian random vector $\boldsymbol{\alpha}=\left\{\alpha_{1}, \ldots, \alpha_{6}\right\}^{T}:=\{\alpha(l), \ldots, \alpha(6 l)\}^{T} \in \mathbb{R}^{6}$ with covariance matrix $\Sigma_{\alpha}:=\left[\mathbb{E}\left(\alpha_{p} \alpha_{q}\right)\right], p, q=1, \ldots, 6$, where $\mathbb{E}\left(\alpha_{p} \alpha_{q}\right)=$ $\mathbb{E}(\alpha(p l) \alpha(q l))=\Gamma_{\alpha}((q-p) l)$, providing complete statistical characterization of spring stiffnesses $k_{i}=c_{\alpha} \exp \left(\alpha_{i}\right)$. Therefore, the input random vector $\boldsymbol{X}=\left\{M, C, K, \alpha_{1}, \ldots, \alpha_{6}\right\}^{T} \in \mathbb{R}^{9}$ includes nine random variables in this example. Further details of the dynamic system, including mass, damping, and stiffness matrices, are given in [8].

Because of nonproportional damping, the discrete beam model yields 14 complex eigenvalues $\lambda_{i}(\boldsymbol{X})=\lambda_{R, i}(\boldsymbol{X}) \pm \sqrt{-1} \lambda_{I, i}(\boldsymbol{X}), i=$ $1, \ldots, 7$ in conjugate pairs, where the real parts $\lambda_{R, i}(\boldsymbol{X})$ and imaginary parts $\lambda_{I, i}(\boldsymbol{X})$ are both stochastic. Figure 5 plots the marginal probability distributions $F_{I, i}\left(\lambda_{I, i}\right)$ and their complementary probabilities $1-F_{I, i}\left(\lambda_{I, i}\right), i=1, \ldots, 7$ of all seven imaginary parts, which also represent the natural frequencies of the beam. The distributions $F_{I, i}\left(\lambda_{I, i}\right)$ and $1-F_{I, i}\left(\lambda_{I, i}\right)$ at low probabilities describe the tail characteristics of $\lambda_{i}$ at the left and right ends, respectively. Each subfigure of Fig. 5 contains four plots: one obtained from crude Monte Carlo simulation and the remaining three generated from the univariate $(S=1)$, bivariate $(S=2)$, and trivariate $(S=3)$ polynomial decomposition methods, employing $m=4$ and $n=5$. The tail probability distributions at both ends converge rapidly with respect to $S$, regardless of the mode. Compared with the Monte Carlo simulation, all three versions of the decomposition method provide satisfactory to excellent estimates of the tail distributions. The bivariate solution, which captures the cooperative effects of any two variables, is remarkably close to the Monte Carlo result. The tail probabilities from the trivariate decomposition and crude Monte Carlo simulation are practically coincident. The probability distributions of the real parts of the eigenvalues reveal the same trend, but they are not reported for brevity.

The univariate, bivariate, and trivariate decomposition methods require 37, 613, and 5989 solutions, respectively, of the matrix characteristic equation, whereas $10^{6}$ (sample size) such solutions are involved in crude Monte Carlo simulation. Clearly, the univariate method is inexpensive, but it is not as accurate as the other two decomposition methods. Nonetheless, all three versions of the decomposition method are far less expensive than crude Monte Carlo simulation.

\section{Example 3: Piezoelectric Transducer}

The final example entails eigenspectrum analysis of a piezoelectric transducer commonly used for converting electrical pulses to mechanical vibrations and vice versa. Figure 6 a shows a 25-mm-diam cylinder made of a piezoelectric ceramic PZT4 (lead zirconate titanate) with brass end caps. The thicknesses of the transducer and end caps are 1.5 and $3 \mathrm{~mm}$, respectively. The cylinder, $25 \mathrm{~mm}$ long, was electroded on both the inner and outer surfaces. The random variables include 1) ten nonzero constants defining elasticity, piezoelectric coupling, and dielectric properties of PZT4, which are described in the Appendix; 2) elastic modulus and

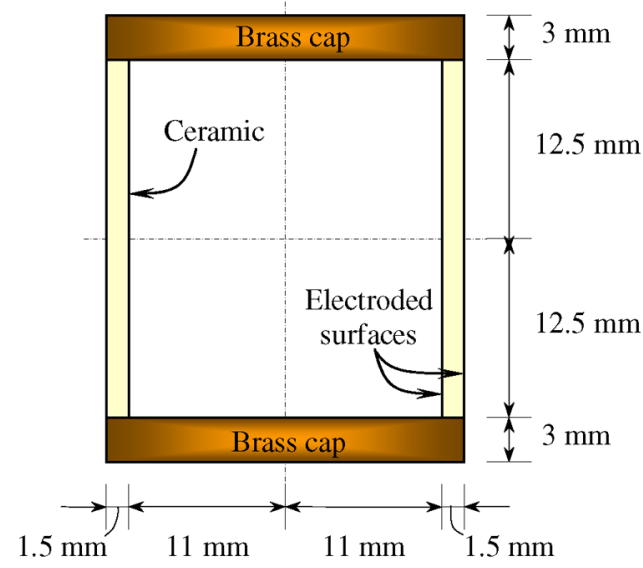

a)

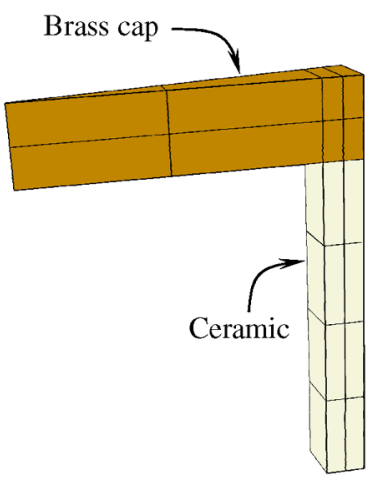

b)

Fig. 6 Piezoelectric transducer: a) geometry, and b) finite element model.

Poisson's ratio of brass; and 3) mass densities of brass and PZT4. The statistical properties of all 14 random variables are listed in Table 2. The random variables are independent and follow lognormal distribütions. Because of axisymmetry, a 20-noded finite element model of a slice of the transducer, shown in Fig. 6b, was created. The model was considered to be open circuited. All natural frequencies calculated correspond to antiresonant frequencies.

In calculating eigenvalues, both deterministic and stochastic analyses were performed. For the deterministic eigenvalue analysis, all random input parameters were fixed at their mean values. Based on the mean input, Fig. 7 portrays the first six mode shapes of the transducer with corresponding natural frequencies of 19.75, 42.90,

Table 2 Statistical properties of random input for piezoelectric cylinder

\begin{tabular}{lccc}
\hline \hline Random variable & Propertya & Mean & Coefficient of variation \\
\hline$X_{1}, \mathrm{GPa}$ & $D_{1111}$ & 115.4 & 0.15 \\
$X_{2}, \mathrm{GPa}$ & $D_{1122}, D_{1133}$ & 74.28 & 0.15 \\
$X_{3}, \mathrm{GPa}$ & $D_{2222}, D_{3333}$ & 139 & 0.15 \\
$X_{4}, \mathrm{GPa}$ & $D_{2233}$ & 77.84 & 0.15 \\
$X_{5}, \mathrm{GPa}$ & $D_{1212}, D_{2323}, D_{1313}$ & 25.64 & 0.15 \\
$X_{6}, \mathrm{C} / \mathrm{m}^{2}$ & $e_{111}$ & 15.08 & 0.1 \\
$X_{7}, \mathrm{C} / \mathrm{m}^{2}$ & $e_{122}, e_{133}$ & -5.207 & 0.1 \\
$X_{8}, \mathrm{C} / \mathrm{m}^{2}$ & $e_{212}, e_{313}$ & 12.71 & 0.1 \\
$X_{9}, \mathrm{nF} / \mathrm{m}$ & $D_{11}$ & 5.872 & 0.1 \\
$X_{10}, \mathrm{nF} / \mathrm{m}$ & $D_{22}, D_{33}$ & 6.752 & 0.1 \\
$X_{11}, \mathrm{GPa}$ & $E_{b}$ & 104 & 0.15 \\
$X_{12}$ & $v_{b}$ & 0.37 & 0.05 \\
$X_{13}, \mathrm{~g} / \mathrm{m}^{3}$ & $\rho_{b}$ & 8500 & 0.15 \\
$X_{14}, \mathrm{~g} / \mathrm{m}^{3}$ & $\rho_{c}$ & 7500 & 0.15 \\
\hline
\end{tabular}

${ }^{\mathrm{a}} D_{i j k l}$ are elastic moduli of ceramic; $e_{i j k}$ are piezoelectric coupling constants of ceramic; $D_{i j}$ are dielectric constants of ceramic; $E_{b}, v_{b}, \rho_{b}$ are elastic modulus, Poisson's ratio, and mass density of brass; $\rho_{c}$ is mass density of ceramic. 

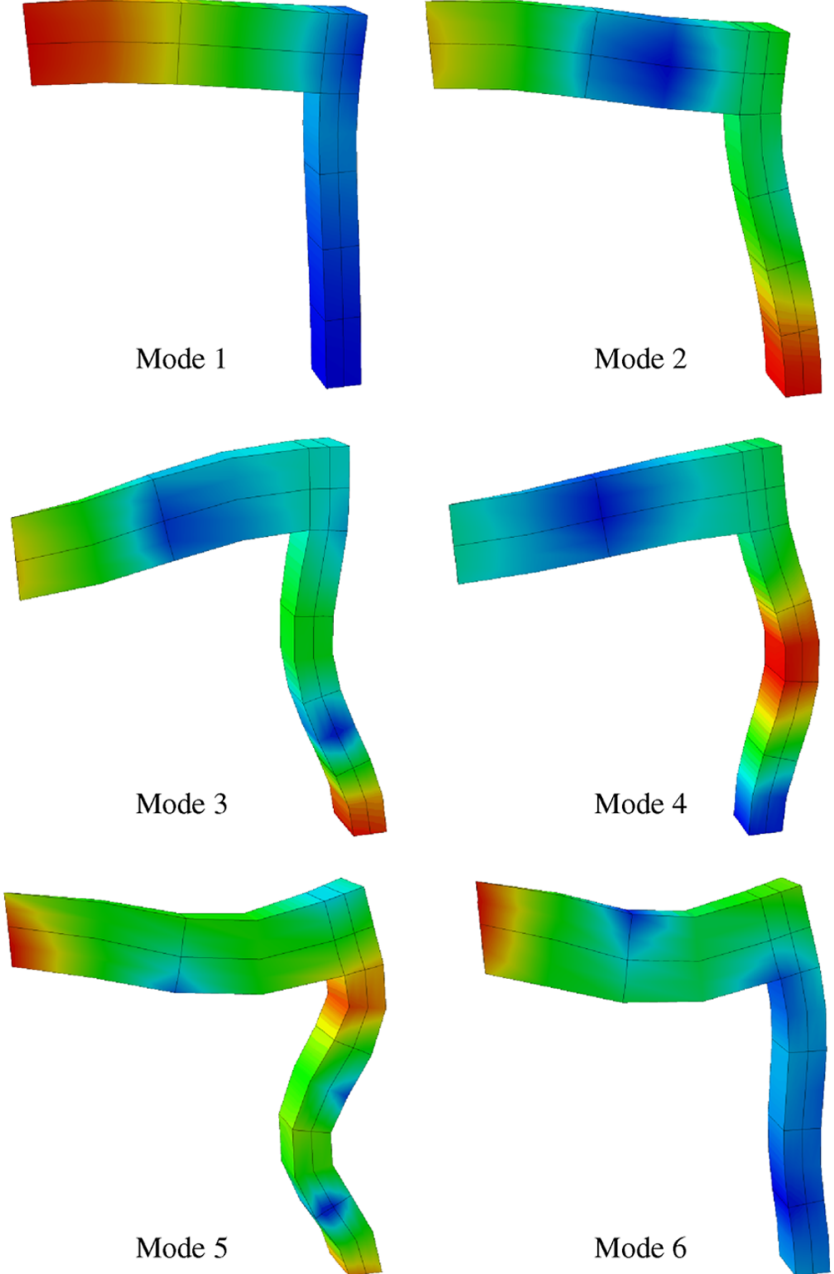

Fig. 7 Mode shapes of a piezoelectric transducer at mean input.

$60.70,66.70,92.03$, and $100.32 \mathrm{kHz}$. These frequencies agree excellently with other numerical results and the experimental data from Mercer et al. [17], both reported in the ABAQUS User's Manual [16].

Figures $8 \mathrm{a}$ and $8 \mathrm{~b}$ present marginal probability densities and distributions, respectively, of the first six natural frequencies, $\Omega_{i}$, $i=1, \ldots, 6$, of the transducer by the univariate and bivariate polynomial decomposition methods. These probabilistic characteristics, obtained by setting $m=3$ and $n=4$, are judged to be converged responses, as their changes due to further increases in $m$ and $n$ are negligibly small. Therefore, only 43 and 862 ABAQUSaided FEA were required in generating all six probability densities or distributions by the univariate and bivariate methods, respectively. Because of expensive FEA, crude Monte Carlo simulation was feasible only up to 5000 realizations, producing only rough estimates of the histograms or cumulative distributions. Given the low sample size, the histograms and distributions, also plotted in Figs. $\underline{8 \mathrm{a}}$ and $\underline{\mathrm{b}}$,

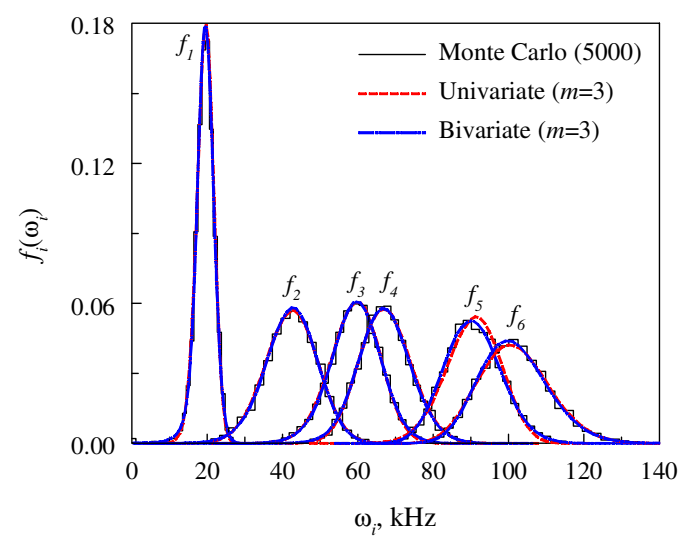

a)

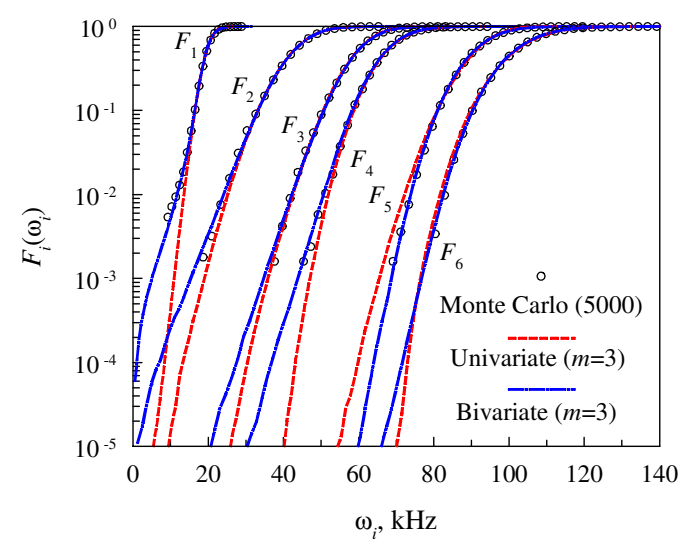

b)

Fig. 8 Marginal probabilistic characteristics of natural frequencies of the piezoelectric transducer by various methods: a) probability densities and histograms, and b) cumulative distribution functions.

respectively, are not expected to provide accurate tail characteristics. Nonetheless, the overall shapes of all six probability densities generated by both decomposition methods match these histograms quite well. For the same reason, the Monte Carlo results are restricted to probabilities approximately equal to or larger than 10/5000. The accuracy of the decomposition method is further verified in Table 3 , which reveals excellent agreement between the second-moment properties of all six frequencies obtained by the decomposition methods and Monte Carlo simulation. This example demonstrates the nonintrusive nature of the proposed stochastic method, which can be easily integrated with external commercial codes.

Finally, Figs. 9a and 9b display the joint probability density function of two arbitrarily chosen frequencies, $\Omega_{3}$ and $\Omega_{4}$, by the bivariate method and crude Monte Carlo simulation, respectively. Although visually comparing two three-dimensional plots is not simple, the joint distributions from both methods agree well. Similar results can be generated by the univariate method or for other natural frequencies if desired.

Table 3 Second-moment properties of natural frequencies of piezoelectric transducer

\begin{tabular}{lcccccc}
\hline \hline & \multicolumn{2}{c}{ Mean } & \multicolumn{3}{c}{ Standard deviation } \\
\hline Frequency & Univariate & Bivariate & Monte Carlo & Univariate & Bivariate & Monte Carlo \\
\hline$\Omega_{1}, \mathrm{kHz}$ & 19.45 & 19.38 & 19.39 & 2.30 & 2.54 & 2.66 \\
$\Omega_{2}, \mathrm{kHz}$ & 42.31 & 42.27 & 42.30 & 7.03 & 7.09 & 7.25 \\
$\Omega_{3}, \mathrm{kHz}$ & 59.23 & 59.42 & 59.35 & 6.65 & 6.82 & 6.94 \\
$\Omega_{4}, \mathrm{kHz}$ & 67.44 & 67.21 & 67.24 & 6.94 & 7.09 & 7.02 \\
$\Omega_{5}, \mathrm{kHz}$ & 90.57 & 90.60 & 90.71 & 7.38 & 7.58 & 7.58 \\
$\Omega_{6}, \mathrm{kHz}$ & 101.69 & 101.66 & 101.72 & 9.43 & 9.24 & 9.29 \\
\hline \hline
\end{tabular}




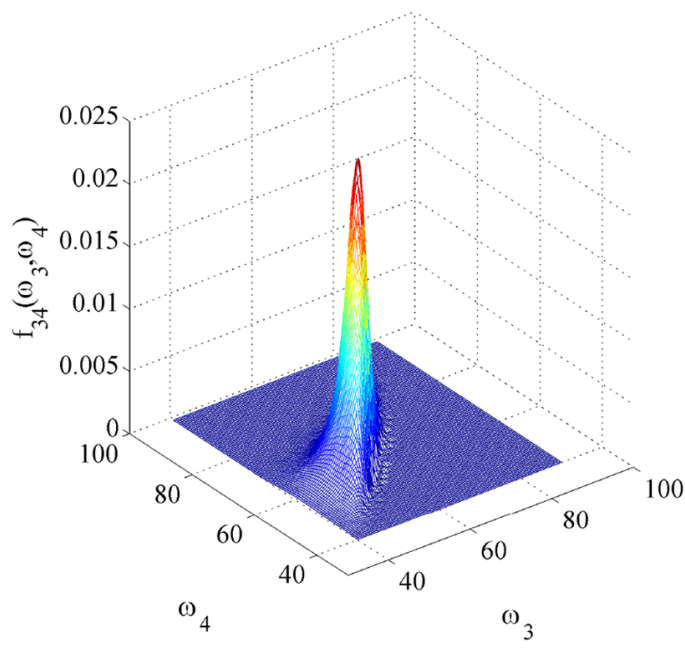

a)

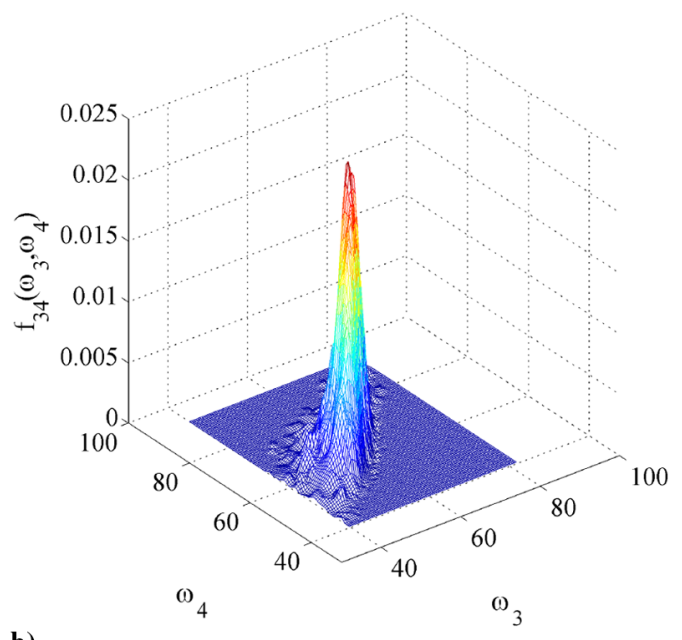

b)

Fig. 9 Joint probability density of third and fourth natural frequencies of piezoelectric transducer: a) bivariate method, and b) crude Monte Carlo simulation.

\section{Conclusions}

A polynomial dimensional decomposition method was developed for calculating the probability distributions of random eigenvalues frequently encountered in dynamic systems. The method is based on a hierarchical decomposition of a multivariate function in terms of variables with increasing dimensions, a broad range of orthonormal polynomial bases consistent with the probability measure for a Fourier-polynomial expansion of component functions, and an innovative dimension-reduction integration for calculating the expansion coefficients. The new decomposition method does not require sample points around the mean input to approximate the component functions. Instead, orthogonal polynomial basis functions in the Hilbert space, such as Hermite polynomials, were employed, yielding the Fourier-polynomial expansion of the component functions. Because of the nonintrusive evaluation of the expansion coefficients, the method can be easily adapted to solving complex stochastic problems requiring external deterministic codes.

Three dynamic systems were analyzed to evaluate the probability distributions of their eigenvalues, including the frequency distributions of a piezoelectric transducer. The results indicate that the polynomial decomposition method developed, in particular the bivariate and trivariate versions, provides accurate and convergent estimates of the tail distributions of eigenvalues. The orders of polynomials required for calculating tail probabilities accurately depend on the computed probability. The computational effort by the univariate method varies linearly with respect to the number of random variables or the number of integration points; therefore, the univariate method is economical. In contrast, the bivariate and trivariate methods, which are generally superior to the univariate method, demand a quadratic or cubic cost scaling, making either method more expensive than the univariate method. Nonetheless, all three versions of the decomposition method are significantly more economical than crude Monte Carlo simulation.

For input random variables with an arbitrary probability measure, independent or dependent, a direct approach to polynomial dimensional decomposition requires constructing measure-consistent nonclassical orthonormal polynomials, including cases in which a random input comprises both discrete and continuous probability measures. Using these nonclassical orthonormal polynomials, one will be able to determine how a choice of transformation commonly employed in the indirect approach affects the smoothness of an eigenvalue function and to evaluate the accuracy and convergence properties of a stochastic solution against those obtained from the direct approach. Although the $S$-variate Fourier-polynomial approximation can be invoked for a discontinuous function (due to the completeness of polynomials in $\mathcal{L}_{2}$ ), its convergence rate may be significantly degraded. Therefore, the decomposition method developed needs to be extended by improving convergence properties for discontinuous or nonsmooth stochastic responses found in some random eigenvalue problems. These are subjects of future research.

\section{Appendix A: Piezoelectric Analysis and System Equations}

The piezoelectric effect is governed by coupled stress and electrical field and requires the solution of the mechanical equilibrium equation

$$
\int_{V} \boldsymbol{\sigma}: \delta \boldsymbol{\epsilon} \mathrm{d} V=\int_{S} \boldsymbol{t} \cdot \delta \boldsymbol{u} \mathrm{d} S+\int_{V} \boldsymbol{f} \cdot \delta \boldsymbol{u} \mathrm{d} V
$$

and the electric flux equation

$$
\int_{V} \boldsymbol{q}: \delta E \mathrm{~d} V=\int_{S} q_{S} \cdot \delta \varphi \mathrm{d} S+\int_{V} q_{V} \cdot \delta \varphi \mathrm{d} V
$$

simultaneously, where $\sigma$ is the Cauchy stress tensor, $t$ is the traction vector with surface area $S, \boldsymbol{f}=-\rho \ddot{\boldsymbol{u}}$ is the d'Alembert force vector with $\rho$ denoting mass density of the body with volume $V, \delta \boldsymbol{u}$ is the virtual displacement, $\delta \boldsymbol{\epsilon}$ is the virtual strain, $\boldsymbol{q}$ is the electric flux (electrical displacement) vector, $q_{S}$ is the electric flux per unit area entering the body, $q_{V}$ is the electric flux per unit volume entering the body, $\delta \varphi$ is the virtual electric potential, and $\delta E$ is the virtual potential gradient (electrical field). The constitutive behavior of the piezoelectric media is described by

$$
\sigma_{i j}=D_{i j k l} \epsilon_{k l}-e_{m i j} E_{m}
$$

and

$$
q_{i}=e_{i j k} \epsilon_{j k}+D_{i j} E_{j}
$$

where $D_{i j k l}, e_{m i j}$, and $D_{i j}$ are the elastic moduli, piezoelectric constants, and dielectric constants, respectively, and the subscripts indicate appropriate components. Further details are available elsewhere [18].

Consider a finite element discretization, where $\boldsymbol{U}$ and $\boldsymbol{\Phi}$ denote vectors of displacements and electric potentials at the nodal locations. Then, the approximate system of matrix equations becomes

$$
\boldsymbol{M} \ddot{\boldsymbol{U}}+\boldsymbol{K}_{u u} \boldsymbol{U}+\boldsymbol{K}_{\varphi u} \boldsymbol{\Phi}=\boldsymbol{P}
$$

and

$$
\boldsymbol{K}_{\varphi u} \boldsymbol{U}-\boldsymbol{K}_{\varphi \varphi} \boldsymbol{\Phi}=-\boldsymbol{Q}
$$

where $\boldsymbol{M}$ is the mass matrix, $\boldsymbol{K}_{u u}$ is the displacement stiffness matrix, $\boldsymbol{K}_{\varphi u}$ is the piezoelectric coupling matrix, $\boldsymbol{K}_{\varphi \varphi}$ is the dielectric stiffness matrix, $\boldsymbol{P}$ is the mechanical force vector, and $\boldsymbol{Q}$ is the electrical 
charge vector. These coefficient matrices, which comprise elastic moduli, coupling constants, and dielectric constants, also depend on the shape function of an element.

\section{Acknowledgment}

The author would like to acknowledge financial support from the U.S. National Science Foundation under grant no. CMMI-0653279.

\section{References}

[1] Boyce, W. E., Probabilistic Methods in Applied Mathematics I, Academic Press, New York, 1968.

[2] Mehlhose, S., Vom Scheidt, J., and Wunderlich, R., "Random Eigenvalue Problems for Bending Vibrations of Beams," Zeitschrift für Angewandte Mathematik und Mechanik, Vol. 79, 1999, pp. 693-702. doi:10.1002/(SICI)1521-4001(199910)79:10<693::AIDZAMM693>3.0.CO;2-0

[3] Grigoriu, M., "A Solution of the Random Eigenvalue Problem by Crossing Theory," Journal of Sound and Vibration, Vol. 158, No. 1, 1992, pp. 69-80. doi:10.1016/0022-460X(92)90664-J

[4] Nair, P. B., and Keane, A. J., "An Approximate Solution Scheme for the Algebraic Random Eigenvalue Problem," Journal of Sound and Vibration, Vol. 260, No. 1, 2003, pp. 45-65. doi:10.1016/S0022-460X(02)00899-4

[5] Adhikari, S., and Friswell, M. I., "Random Matrix Eigenvalue Problems in Structural Dynamics," International Journal for Numerical Methods in Engineering, Vol. 69, 2007, pp. 562-591. doi:10.1002/nme.1781

[6] Ghosh, D., Ghanem, R. G., and Red-Horse, J., "Analysis of Eigenvalues and Modal Interaction of Stochastic Systems," AIAA Journal, Vol. 43, 2005, pp. 2196-2201. doi: $10.2514 / 1.8786$

[7] Rahman, S., "A Solution of the Random Eigenvalue Problem by a Dimensional Decomposition Method," International Journal for Numerical Methods in Engineering, Vol. 67, 2006, pp. 1318-1340. doi:10.1002/nme.1665

[8] Rahman, S., "Stochastic Dynamic Systems with Complex-Valued
Eigensolutions," International Journal for Numerical Methods in Engineering, Vol. 71, 2007, pp. 963-986. doi:10.1002/nme.1973

[9] Efron, B., and Stein, C., "The Jackknife Estimate of Variance," Annals of Statistics, Vol. 9, 1981, pp. 586-596. doi:10.1214/aos/1176345462

[10] Rabitz, H., and Alis, O., "General Foundations of High Dimensional Model Representations," Journal of Mathematical Chemistry, Vol. 25, 1999, pp. 197-233. doi:10.1023/A:1019188517934

[11] Sobol, I. M., "Theorems and Examples on High Dimensional Model Representations," Reliability Engineering and System Safety, Vol. 79 , No. 2, Feb. 2003, pp. 187-193.doi:10.1016/S0951-8320(02)00229-6

[12] Rahman, S., "A Polynomial Dimensional Decomposition for Stochastic Computing," International Journal for Numerical Methods in Engineering, Vol. 76, 2008, pp. 2091-2116. doi: $10.1002 / \mathrm{nme} .2394$

[13] Xu, H., and Rahman, S., "A Generalized Dimension-Reduction Method for Multi-Dimensional Integration in Stochastic Mechanics," International Journal for Numerical Methods in Engineering, Vol. 61, 2004, pp. 1992-2019. doi:10.1002/nme.1135

[14] Abramowitz, M., and Stegun, I. A., Handbook of Mathematical Functions, 9th ed., Dover, New York, 1972.

[15] International Mathematics and Statistics Library Numerical Libraries, User's Guide and Theoretical Manual, Visual Numerics, Inc., San Ramon, CA, 2005.

[16] ABAQUS, User's Guide and Theoretical Manual, Ver. 6.8, ABAQUS, Inc., Providence, RI, 2008.

[17] Mercer, C. D., Reddy, B. D., and Eve, R. A., "Finite Element Method for Piezoelectric Media," Univ. of Cape Town, Council for Scientific and Industrial Research Applied Mechanics Research Unit, Technical Rept. 92, Cape Town, South Africa, 1987.

[18] Ikeda, T., Fundamentals of Piezoelectricity, Oxford Science Press, Oxford, 1990. 